\title{
Problems in the collection and analysis of data in leprosy studies
}

\author{
P E M FINE \\ Ross Institute, London School of Hygiene and Tropical Medicine, Keppel \\ Street, London EC1, England
}

Leprosy is perhaps the least well understood of all the major infectious diseases of man. There is an irony in this, in that Mycobacterium leprae was the first bacterial pathogen of man to be recognized. But we still do not know the growth requirements of the organism; we do not understand the varied responses of the host to infection; and even the sources and modes of transmission are subjects of controversy. Our ignorance is only partly attributable to the relative neglect of leprosy by medical researchers in the past. This neglect was itself - at least to some extent - a reflection of the formidable difficulties in studying this disease. Few people enjoy bruising their heads on stone walls. Recent advances in the in vivo propagation of $M$. leprae, and in relevant aspects of basic immunology, have challenged the long stalemate; but these findings have yet to be carried into the field to unravel fully the natural history of this disease. Inherent difficulties remain.

This paper reviews some of the problems which arise in epidemiological studies of leprosy. They can be considered as three groups of problems: of case definition, of data collection and of data analysis and interpretation. Of course analogous difficulties arise in studies of any disease; but it may be useful to comment on some of the particular problems raised by leprosy.

Defining what are dealing with

What is leprosy? This is more than a rhetorical question. Indeed, case definition is a fundamental first step in any epidemiological exercise. Is leprosy an infection or a disease...; how is it recognized...; how is it classified?

Though most of us recognize the distinction between infection and disease, there is an unfortunate tradition of using the terms interchangeably - as in the phrase "disease transmission". But diseases are not transmitted..., infections are. In equating the terms we lose sight of the fact that the population patterns of each may be very different. Given the length of the incubation period preceding clinical leprosy, and the increasing evidence for large 
numbers of abortive or subclinical infections, it is possible that infected individuals may outnumber clinical cases by as many as 10 or 20 fold in a community. It is the pattern of these infections which holds the key to the natural history of $\mathrm{M}$. leprae. The extent to which infection with $\mathbf{M}$. leprae is not sufficient to cause clinical disease will force us to consider combinations of risk factors for exposure and infection on the one hand and for clinical expression on the other - in interpreting patterns of disease. It was tuberculin which revealed the natural history of M. tuberculosis. Recent studies with lymphocyte transformation tests (1), skin testing with $M$. leprae antigens (2) and in particular Abe's FLA-ABS test (3) allow some optimism that we may soon have a sensitive, specific and fieldworthy test for $M$. leprae infection. Let us hope so - it would be a most important advance towards understanding the epidemiology of leprosy. But until then, we must be satisfied with inferences based on clinical cases and their distribution.

Most clinical leprosy comes to our attention through the recognition of certain skin or nerve lesions. Most cases are screened first - if not only - by paramedically trained auxiliaries. Some cases are referred for medical attention, and a smaller proportion are subjected to smears, biopsy or lepromin tests to assist in diagnosis and classification. All this information is sifted, and then out drop "cases" into neat boxes labelled according to Indian, Madrid or Ridley-Jopling categories, depending on local prejudices. To read the literature one might think this sifting a simple exercise, and the categories inviolate. On the other hand, most of us are aware that the diagnosis of leprosy is often a difficult matter, that its classification is controversial, and that we face problems of comparability between case series from different regions and workers. Newell (4) commented succinctly in 1966: "It is exceptional, rather than normal, for the criteria for inclusion or exclusion to be stated in leprosy publications. This fact casts doubts upon many of the painstaking and time-consuming community studies which have been done and makes accurate comparison impossible".

The situation is not much better today, except to the extent that one increasingly reads that cases were biopsied and classified according to the Ridley-Jopling system. The Ridley-Jopling scale (5) does represent an important step towards placing the diagnosis of leprosy on an objective basis. Still, this phrase belies a number of difficulties: what were the criteria for a biopsy to be taken in the first place; what was done when the biopsy report clashed with clinical impression; how much clinical information was provided to the histopathologist; what was done when the biopsy report implied some uncertainty in the diagnosis or classification? My experience tells me that each of these issues arises frequently and may have an important influence on ultimate classification of cases - though I know of no study which examines them carefully.

The case definition problem is probably most obvious with respect to the use of an indeterminate category. I am aware that major differences of opinion exist between participants of this 
meeting, as to the criteria for and prevalence of "indeterminate" leprosy. This is not a trivial matter. For example, important immunological studies are currently underway in South America on what are called indeterminate cases. It is important that the criteria for this classification be unambiguous if these results are to be transferrable.

Surely among the interesting epidemiological puzzles in leprosy are the apparent regional and ethnic differences in its clinical manifestation. To what extent these may be due to differences in skin pigmentation, to methods of ascertainment of cases, to regional clinical diagnostic prejudices or to the training of histopathologists is unclear. In order to ensure comparability between research in different areas of the world, and insofar as the answer may point to important variables in the natural history of leprosy, this question is worth answering.

I have been asked to comment upon appropriate classification for epidemiological work, and whether the Ridley-Jopling system should be simplified to this end. Being neither clinician nor histopathologist, I hesitate to go too far out on this limb, but would at least suggest a set of criteria against which to measure classification systems. i) The system should be clear, expressed in explicit terms. ii) It should be objective, affected as little as possible by subjective experience or clinical impressions of the observers. iii) It should make sense clinically, histopathologically and immunologically. iv) It should be practicable in the hands of many persons. This of course means that different systems may be appropriate for large-scale survey work on the one hand and for limited intensive studies on the other. v) It should be as detailed as possible within the above constraints. Of course none of these criteria is original, and some would claim that the Madrid and Ridley-Jopling systems satisfy them. But there are three additional points. vi) The system should be fully standardized regarding the clinical features to be taken into consideration, the criteria and method for biopsy or immunological test, the amount of clinical information supplied to the histopathologist, and the interpretation of apparent conflicts between clinical, lepromin, biopsy etc. information. vii) The system should be assessed for comparability in different hands. This means more than correlations between clinician and histopathologist, as have been carried out (e.g. 6) but proper blind comparisons between different observers asked to examine the same material. Such comparability studies should include workers from different traditions. viii) Finally, the system should be acceptable, and accepted. Easier said than done, perhaps, but without acceptance the value of any classification is greatly compromised. We must agree on our dictionary.

Collection of data

In general, information on leprosy comes to us from three sources: 
routine health services data; population-based surveys; and special studies directed at small groups of individuals.

ROUTINE DATA: Information on leprosy is contained in national or regional leprosy registers, notification schemes, hospital inor out-patient statistics and treatment records of domiciliary control programmes. The quantity of such information varies greatly between different areas of the world, but its quality is in general poor. This in part reflects the unfortunate concentration of leprosy in those countries, districts and population groups with the least developed health serrices. Most routine data relate to passively detected cases, supplemented in some circumstances by limited active case finding - either household contact tracing or school surveys or house-to-house surveys. One of the major problems with such data is the fact that the intensity and methods of ascertainment, let alone methods of diagnosis and classification, are typically unclear. And without such background information, the interpretation of such data is difficult.

Numbers of cases are largely a function of how hard and how often you look. We know that a considerable proportion - up to $80 \%$ (7)? - of cases may resolve completely. Most of these will not self-report. We learn about them only through aggressive case finding activities. The milder self-healing forms are generally towards the tuberculoid end of the spectrum; and hence poor case finding will both underestimate prevalence and distort the type distribution of cases towards the lepromatous pole. As prevalence is a function of incidence and duration, the failure to remove cured cases will tend to inflate the apparent tuberculoid case load disproportionately. And of course the peculiar stigma of leprosy, and its socioeconomic distribution, will often have the effect of selectively hiding cases from attention.

In general, I would take the pessimistic view that without detailed information on methods of case finding, diagnostic practices and maintenance of registers (in particular the removal of cases after death or cure), such routine statistics are of little use beyond the mere indication that leprosy exists in an area. The literature devoted to these statistics is - with one important exception mentioned below - disappointing owing to poor data quality. The considerable effort which has gone to compile such statistics on a world wide basis may assist in fund-raising or in justifying the persistence of some institutions; but I question whether it contributes to our understanding of the disease.

The important exception to this critique of routine deprosy data is of course the Norwegian Leprosy Register. This is indisputably a valuable resource, and Irgens' recent monograph (8) is a most useful document, full of insights. But this situation is unique in its status as a total national register of long duration. Furthermore, its value was greatly enhanced by the availability of detailed historical census, meteorological and tax information against which to analyze the Register's data. I am aware of no other comparable situation. 
POPULATION-BASED SURVEYS: The first population-based studies of leprosy were those begun by Doull in the Philippines, almost 50 years ago (e.g.9). It is interesting to note that Doull was a protege of Wade Hampton Frost, the greatest figure in American epidemiology, and that he was in effect applying techniques developed by Frost in studies of tuberculosis. In terms of conception, organization and presentation, this work of Doull still stands unrivalled in the leprosy literature. Relatively little has been added to his descriptions of the patterns of leprosy prevalence and of the risks associated with household contact with lepromatous or tuberculoid cases.

Few total population studies have been carried out since Doull's efforts, with notable exception of house-to-house surveys associated with control programmes as in Karigiri, Chingleput and Polambakkam in South India (e.g.10). This is not surprising, as the low prevalence of leprosy makes such studies prohibitively expensive in time, cost and personnel, and raises major problems in field logistics, supervision and data handling. With total prevalence rates on the order of $1 \%$, and much lower for lepromatous disease, it is necessary to examine many tens of thousands of persons to obtain a detailed picture of leprosy prevalence. The considerable investment in such work means that great care should be taken in early planning stages. Several large data sets exist which are effectively unusable, because their research potential, the need for documentation and continuity, and the practical problems of handling large amounts of data were not recognized early. For such reasons most epidemiological survey work in recent years has been carried out as an active case finding phase of a control programme, or as baseline studies for trials of BCG or chemoprophylaxis (e.g. 11).

The well-recognized familial and village clustering of leprosy raises further problems for survey design. Combined with the low prevalence of leprosy such clustering may make true random sampling surveys (e.g.12) difficult if not inappropriate. Cluster sampling is more efficient of resources but may be misleading if the aim is to describe the pattern of leprosy in an area. Similarly, selective surveys of household "contacts" or of schoolchildren may be efficient as case finding techniques but do not provide a full or accurate picture of leprosy in the community. The widespread a priori belief in clustering of leprosy raises another problem in that field workers are liable to alter their index of suspicion and look more intensively at household members once a first case has been recognized. This bias - which in effect makes household clustering a self-fulfilling prophecy - is very difficult to avoid.

Such problems are compunded in efforts at longitudinal studies of incidence. Incidence rates of 1 per 1000 per year necessitate very large study populations and long follow-up periods, and are not to be undertaken lightly. If interest is primarily in incidence of lepromatous disease - as it may be, for example, in a vac- 
cine trial - then these practical difficulties are even greater. Losses to follow-up are liable to be numerous in such studies, and may include disproportionate numbers of leprosy cases, constituting an important bias. Doull (9) was surprisingly successful in estimating incidence patterns on the basis of historical data collected in a single survey; but his success was largely due to his superbly critical analysis and this method is not to be encouraged. Our best recent longitudinal data have been collected in the major controlled trials of BCG, and constitute an important argument in favour of such trials.

SPECIAL STUDIES: The great cost and practical difficulties associated with population-based studies of leprosy encourage us to examine the feasibility of studying selected groups. Some studies of chemotherapeutic, surgical, immunological or histopathological aspects of leprosy require only limited numbers of patients. Here the major concern is that the investigated cases be correctly classified and representative of the groups under discussion. If controls are required, as in the screening of immunological tests, it is important that they be wisely chosen and carefully documented for example with regard to age, history of BCG or evidence of any mycobacterial exposure, infection or disease.

More interesting methodological problems arise in efforts to assess risk factors for infection, disease, reactions, defaulting, etc. It is notable that most of the factors known to be associated with a high risk of clinical leprosy (age, sex, BCG history, tuberculin and lepromin sensitivity, and household contact especially with lepromatous cases) have been established through prospective incidence studies. But an epidemiologist, in surveying the leprosy literature is struck by the absence of case-control studies except insofar as case-control logic is inherent in the twin, genetic marker and familial segregation studies which have revealed an HLA-associated risk factor in leprosy (13). By casecontrol study we mean an investigation which aims at comparing the frequency of some putative risk factor in a group of cases with that in an appropriately chosen control series (14). Such methods are especially appropriate for rare diseases such as leprosy, and they can provide measures of relative (though not absolute) risk associated with such factors. (A relative risk is analogous to Doull's (9) estimate, based on a ratio of incidence rates, that household contacts of cutaneous leprosy cases were approximately 8 times more likely to contract leprosy than were persons not known to have household contact with leprosy).

Two reasons probably explain the absence of these methods from the leprosy literature. One is the separation of leprosy from mainstream epidemiology - unfortunately, as many of the methods evolved in recent years for studies of chronic diseases, in particular cancer, are applicable to leprosy. Other reasons are the practical difficulties associated with leprosy due to its long incubation period, disrupting social effects and prevalence in cultures in which it may be difficult to obtain reliable information concern- 
ing the past. It appears to me, however, that such methods could be applied to assess some of the factors sometimes claimed to be associated with leprosy risk - such as crowding, hygiene practices, diet, clothing, tuberculosis, atypical mycobacteria, type and duration of exposure to cases, bare feet, pregnancy, breast feeding, etc. Extreme care must be taken in selecting controls for such studies, as many of the hypothesized factors confound one another as part of the poverty complex. One may need to look within families in order to match for genetical and contact factors. (Note the danger in using hospital staff as controls in immunological or genetical studies - this may mislead more than enlighten). But there seems to be an opening here for productive work by careful and critical epidemiologists.

\section{Analysis and interpretation of data}

Few recent authors have approached the high standards set by Doull for the careful analysis of leprosy data. There is at least an increasing tendency to recognize the distinction between incidence and prevalence, to use person-years denominators and to apply standard statistical tests; but it is not unusual for authors to make too much, or too little, of their data. Many of the analysis problems concern interactions between variables.

In analyzing immunological measures, for example, it must be recognized that sensitivity to mycobacterial skin test antigens, lepromin positivity, BCG history and leprosy incidence are all strongly age-related. Spurious correlations can arise - or actual correlations may be lost - if this is not taken into account. A nice example of this is seen in the Uganda BCG trial analyses, in which a consistent linear correlation between initial tuberculin sensitivity and leprosy incidence was only revealed after age-standardization of the data (15).

Leprosy appears to be associated with poverty, and this has led to hypotheses that crowding, poor hygiene, skin contact, poor nutrition or intercurrent illness might be important risk factors. The disentanglement of so many strongly correlated variables presents a considerable challenge in collection as well as analysis of data.

There is a temptation to infer risks from cross-sectional data. This can be dangerous, as current condition - for example living conditions - of leprosy patients may reflect the effects rather than "causes" of leprosy. The long incubation period of leprosy further weakens such arguments; as the current state of patients may have little bearing on their situation many years previously when infection occurred. In considering such associations, it is useful to bear in mind that there may be very different risk factors associated with infection on the one hand and with disease on the other. Nevertheless, it is useful to recognize the possibilities of inferring incidence from age specific prevalence data. This is possible on the basis of assumptions covering trends in incidence 
in the past, differential mortality and cure rates. Doull (9) used such an approach in his argument that higher prevalence rates of leprosy among males are attributable to higher incidence. These methods have been elegantly developed in the context of studies of tuberculin sensitivity data, especially by Sutherland (16). If extensive immuno-epidemiological data become available for leprosy, we could again call upon the tuberculosis literature for guidance.

One of the most important epidemiological questions in leprosy concerns the source of infection and the reason for case patterns in the community. This often leads to discussions concerning relationships and contact patterns between known cases. We should be aware of the problems in such arguments. We have dealt with cases because we have had to, as there was no measure of infection. But icebergs may have peculiar shapes below the surface. The word "contact" is generally used in the leprosy literature for coexistent cases in the same household. To the extent that contact implies a source of infection this may be misleading, as infection may occur many years before disease onset when household structure was very different from that observed. Furthermore, the intimacy of contact between individuals is strongly confounded by genetic relationship, and these two influences may be very difficult to untangle (17). The labelling of household cases as "contacts" and the concept of "prolonged and intimate contact" which so haunt the leprosy literature deepen the conspiracy. Several studies have shown that incidence rates are indeed much higher among household "contacts" of multibacillary cases than among any other group. But the rarity of multibacillary cases in many endemic communities means that most new leprosy cases have no apparent connection with the known bacilliferous cases. This leads to two alternatives: either much M. leprae transmission is attributable to individuals whom we do not recognize as bacilliferous cases; or transmission does originate chiefly from bacilliferous cases but it requires only minimal - and not prolonged or intimate - contact. The resolution of this puzzle is an important challenge facing us all.

\section{Conclusions}

Leprosy is sometimes defined as a chronic disease affecting skin and nerves and attributable to Mycobacterium leprae. It might also be described as a rare disease of varied and controversial manifestations, found mainly in poor and distant populations, and characterized by ostracism of cases, fear in contacts, neglect by administrators, hyperbole in fund-raisers, a blind eye in the medical establishment and dogma among its committed workers. There in lie many of the problems in its study. The following suggestions may help us to surmount some of them:

i. A major international collaborative stuảy should be encouraged to assess comparability of diagnosis and classification between different 
clinicians and histopathologists.

ii. Publications should be fully explicit on procedures for diagnosis and classification of cases.

iii. Greater emphasis should be placed upon improving and documenting field ascertainment methods than on institutionalizing poor routine data.

iv. Immunological measures of infection should be applied in population-based studies of the epidemiology of $\underline{M}$. leprae infection.

v. Case control methods should be applied to studies of risk factors for infection and for disease.

\section{Acknowledgements}

The author is grateful for many long and stimulating discussions with Dr Jorg Ponnighaus.

\section{$\underline{\text { References }}$}

1 Godal T, Negassi K. Subclinical infection in leprosy. Brit Med J, 1973, iii, 557-559.

2 Convit J, Pinardi ME, Rojas FA, Gonzales I, Corey G, Arvelo JJ, Monzon $\mathrm{H}$. Tests with three antigens in leprosy-endemic and non-endemic areas. Bull wld Hlth Org, 1975, 52, 193-198.

3 Abe M, Minagawa F, Yoshino Y, Ozawa T, Saikawa K, Saito T. Fluorescent antibody absorption (FLA-ABS) test for detecting subclinical infection with Mycobacterium leprae. Int J Lepr, $1980,48,109-119$.

4 Newell $\mathrm{KW}$. An epidemiologist's view of leprosy. Bull Wld Hlth Org, 1966, 34, 827-857.

5 Ridley DS, Jopling WH. Classification of leprosy according to immunity. A five-group system. Int J Lepr, 1966, 34, 255-273.

6 Meyers WM, Heggie CD, Kay TL, Staple EM, Kvernes S. The RidleyJopling five-group classification of leprosy-correlations of parameters of the classification in 1429 leprosy patients. Int J Lepr, 1979, 47, 683-684.

7 Lara CB, Nolasco JO. Self-healing, or abortive, and residual forms of childhood leprosy and their probable significance. Int J Lepr, 1956, 24, 245-263.

8 Irgens LM. Leprosy in Norway: an epidemiological study based on a national patient registry. Lepr Rev, 1980, 51, suppl 1, 1-130.

9 Doull JA, Guinto RS, Rodriguez JN, Bancroft H. The incidence of leprosy in Cordova and Talisay, Cebu, Philippines. 
Int J Lepr, 1942, 10, 107-131.

10 Rao PSS, Karat ABA, Kaliaperumal VG, Karat S. Incidence of leprosy in Gudiyatham Taluk, South Sindia. Ind J Med Res, 1972 , 60, 97-105.

11 Bechelli LM, Gallego Garbajoso P, Gyi MM, Uemura K, Sundaresan, Tamondong, Martinez Dominguez V, Walker J. Some epidemiological data on leprosy collected in a mass survey in Burma. Bull Wld Hlth Org, 1.973, 48, 335-344.

12 World Health Organization. WHO Expert Committee on Leprosy, Fifth Report. Wld Hlth Org Tech Rep Ser, 607, 1977.

13 Fine PEM. Immunogenetics of susceptibility to leprosy, tuberculosis and leishmaniasis. Lepr Rev,1981, 52, (in press).

14 MacMahon B, Pugh TF. Epidemiology: Principles and Methods. Boston: Little, Brown and Company, 1970.

15 Brown JAK, Stone MM, Sutherland I. B.C.G. vaccination of children against leprosy in Uganda: results at end of second follow up. Brit Med J, 1968, i, 24-27.

16 Sutherland I. Recent studies in the epidemiology of tuberculosis, based on the risk of being infected with tubercle bacilli. Adv Tuberc Res, 1976, 19, 1-63.

17 White SJ, Stone MM, Howland C. Genetic factors in leprosy: a study of children in Uganda. J Hyg Camb, 1978, 80, 205-216. 